\title{
Antioxidant support to ameliorate the oxaliplatin-dependent microglial alteration: Morphological and molecular study
}

\author{
Jacopo J.V. Branca, ${ }^{1}$ Donatello Carrino, ${ }^{1}$ Ferdinando Paternostro, ${ }^{1}$ Massimo Gulisano, ${ }^{1}$ Matteo Becatti, ${ }^{2}$ \\ Lorenzo Di Cesare Mannelli, ${ }^{3}$ Alessandra Pacini ${ }^{1}$ \\ ${ }^{1}$ Department of Experimental and Clinical Medicine, Anatomy Section \\ ${ }^{2}$ Department of Experimental and Clinical Biomedical Sciences "Mario Serio" \\ ${ }^{3}$ Department of Neuroscience, Psychology, Drug Research and Child Health (NEUROFARBA), Pharmacology and \\ Toxicology Section, University of Florence, Italy
}

Oxaliplatin is a third-generation chemotherapy drug mainly used for colorectal cancer treatment. However, it is also known to trigger neuropathy whose underlying neurobiological mechanisms are still under investigation and currently available treatments show limited efficacy. It is now established that neurons are not the only cell type involved in chronic pain and that glial cells, mainly microglia and astrocytes, are implicated in the initiation and maintenance of neuropathy. Among all the pathogenetic factors involved in neuropathic pain, an oxaliplatin-dependent oxidative stress plays a predominant role. In our study, the antioxidant properties of magnesium $(\mathrm{Mg})$, manganese $(\mathrm{Mn})$ and zinc $(\mathrm{Zn})$ salts were evaluated in order to counteract microglial activation induced by oxaliplatin. The antioxidant efficacy of these metals was evaluated by means of molecular and morphological assays on the BV-2 microglial cell line. Our data clearly show that $\mathrm{Mg}, \mathrm{Mn}$ and $\mathrm{Zn}$ salts are able to prevent oxaliplatin-dependent microglial alterations by reducing both oxidative and endoplasmic reticulum stress.

Key words: Neuropathic pain; oxaliplatin; magnesium; manganese; zinc.

Correspondence: Prof. Alessandra Pacini, Department of Experimental and Clinical Medicine, Anatomy Section, University of Florence, Largo Brambilla 3, 50134 Florence, Italy.

Tel.+39.055.2758065. E- mail: alessandra.pacini@unifi.it

Contributions: Conception of the work (LDCM and AP); analysis and data interpretation (JJVB, DC and MB); drafting the work (JJVB and AP); critically revision of the work (FP and MG). 


\section{Introduction}

The incidence of tumour burden, nowadays, is still growing especially in the developing countries, mainly due to population growth and ageing, as previously predicted. ${ }^{1}$ However, thanks to the scientific and technological advances, prevention and therapeutic efforts are able to decrease this deleterious pathology, at least in order to improve the quality of life of patients. Among the different cancer types, colorectal cancer is listed as third and second, respectively for incidence and mortality, in both genders worldwide. ${ }^{2}$ Oxaliplatin is the most widely used drugs to counteract colorectal cancer progression, ${ }^{3}$ a third-generation platinumderived chemotherapy agent that has exceeded the cisplatin usage in term of efficacy and cancer resistance. ${ }^{4}$

Despite oxaliplatin anti-cancer efficacy, its side effects, first of all neuropathic pain, constitute a growing field of investigation. It has been reported that oxaliplatin is able to induce glial activation in the central nervous system (CNS), both in spinal cord and in different brain areas. ${ }^{5,6}$ Moreover, recent studies take into account that plasmatic levels of the drug can alter the blood-brain barrier, directly affecting the $\mathrm{CNS}^{7}$ In the past years, many efforts have been made in order to mitigate the oxaliplatin-induced neuropathic pain and the properties of microelements such as magnesium $(\mathrm{Mg})$, manganese $(\mathrm{Mn})$ and zinc $(\mathrm{Zn})$ were investigated. ${ }^{8-10}$

In this study the protective properties of these three microelements, against the oxaliplatin-dependent neurotoxic effects in a microglial cell line, were evaluated.

\section{Materials and Methods}

\section{Cell lines and treatments}

The murine microglia cell line (BV-2), purchased by Istituto Zooprofilattico Sperimentale della Lombardia e dell'Emilia Romagna (Brescia, Italy), was routinely cultured as previously reported. ${ }^{11}$ During each treatment, the medium was replaced with starvation medium (without FBS) with the appropriate stimuli. Oxaliplatin, dissolved in distilled water, was used at $3 \mu \mathrm{M}$ final concentration. Magnesium chloride $\left(\mathrm{MgCl}_{2}\right) 1 \mathrm{mM}$, manganese chloride $\left(\mathrm{MnCl}_{2}\right) 50 \mathrm{nM}$ or zinc chloride $\left(\mathrm{ZnCl}_{2}\right) 100 \mathrm{nM} 1 \mathrm{mM}$ Magnesium chloride $\left(\mathrm{MgCl}_{2}\right)$, or $50 \mathrm{nM}$ manganese chloride $\left(\mathrm{MnCl}_{2}\right)$, or $100 \mathrm{nM}$ zinc chloride $\left(\mathrm{ZnCl}_{2}\right)$ (Sigma Aldrich, Milan, Italy), dissolved in distilled water, was added in the starvation medium $24 \mathrm{~h}$ before and during oxaliplatin treatment, for a total of $48 \mathrm{~h}$.

\section{MTT assay}

BV-2 cells were plated into 96 multiwells at the density of $5 \times 10^{3}$ cells/well, in growth medium. The following day, the cells were treated with increasing concentration of oxaliplatin for $24 \mathrm{~h}$ and a standard procedure for cell viability evaluation was performed as previously reported. ${ }^{7}$

\section{Western blotting analysis}

The BV-2 cells were seeded at $4 \times 10^{6}$ density in Petri dishes ( $\varnothing 100 \mathrm{~mm}$ ) with complete growth medium. The following days, the medium was replaced with starvation medium supplemented with different treatments.

After stimulation, the cells were harvested following a previously used standard procedure for protein extraction, protein quantification and Western blotting analysis. ${ }^{12}$ The specific primary antibody anti-GRP78 (ThermoFischer Scientific, Milan, Italy) was incubated overnight at $4^{\circ} \mathrm{C}$.

\section{Reactive oxygen species (ROS) production analysis}

The BV-2 cells were seeded in 96 multiwells in complete growth medium. The following days, the cells were treated in starvation medium and, after stimulation, the medium was removed and the cells were loaded with $5 \mu \mathrm{M}$ CM- ${ }_{2}$ DCFDA (Life Technologies, ThermoFisher Scientific, Milan, Italy) as previously reported. ${ }^{13}$

\section{Immunofluorescence staining analysis}

The microglial BV-2 cell line was seeded at $1.2 \times 10^{4}$ density on coverslip properly lodge in 6 multiwells in their complete growth medium. The following day, the medium was replaced with starvation medium supplemented with appropriate stimuli. After each treatment, the medium was discarded and a previously used standard procedure was followed. ${ }^{12}$ The primary antibody (rabbit anti-cytochrome $\mathrm{C}$, rabbit anti-Iba1, rabbit anti-Nrf2, or mouse anti-CD86; SantaCruz Biotechnology, Santa Cruz, CA, USA), diluted 1:200 in blocking solution were used. The images were acquired by a motorized 654 Leica DM6000 B microscope equipped with a DFC350FX camera (Leica, Mannheim, Germany).

\section{Statistical analysis}

Statistical analysis was performed by one-way ANOVA followed by the Tukey post-hoc test. All assessments were made by researchers blinded to treatments. Data were analyzed using "Origin 9" software (OriginLab, Northampton, MA, USA). Differences were considered significant at $\mathrm{p}<0.05$.

\section{Results}

\section{MTT assay}

The MTT assay (Figure 1), displayed that oxaliplatin induced a decrease in cell viability in a dose-dependent manner. Accordingly to previously reported data, ${ }^{14}$ we chose an oxaliplatin concentration of $3 \mu \mathrm{M}$ to carry out all the subsequent experiments.

\section{ROS production analysis}

The treatment with oxaliplatin significantly increased the ROS production in comparison to control cells (Figure 2A). On the contrary, the presence of $\mathrm{Mg}, \mathrm{Mn}$ or $\mathrm{Zn}$, counteracted the effect of the chemotherapy.

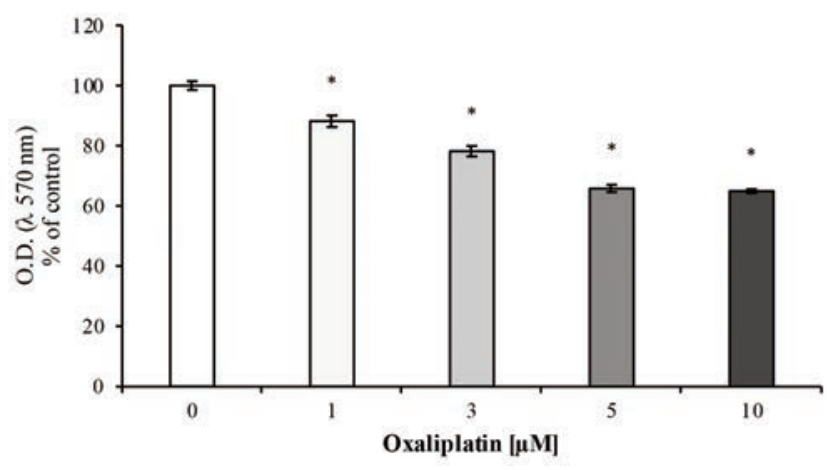

Figure 1. Cell viability assay. Oxaliplatin significantly decrease the cell viability after $24 \mathrm{~h}$ treatment in a dose-dependent manner. Values are expressed in percentage of control (untreated cells) as mean $\pm \mathrm{SEM} ;{ }^{*} \mathrm{p}<0.05$ vs control. Each experiment was performed in quintuplicate, for three times. 


\section{Cytochrome $\mathrm{C}$ analysis}

Since oxaliplatin induces the release of mitochondrial cytochrome $\mathrm{C}$ in astrocytes, ${ }^{15}$ we evaluated the cytoplasmic localization of this protein in BV-2 cells. The presence of oxaliplatin induces an increase in the cytoplasmic localization of the cytochrome $\mathrm{C}$ (black column), whereas the presence of $\mathrm{Mg}, \mathrm{Mn}$, or $\mathrm{Zn}$ was able to counteract this effect (grey columns) (Figure 2B).

\section{Nrf2 nuclear translocation}

Figure $2 \mathrm{C}$ shows that the treatment with microelements was able to prevent the Nrf2 nuclear translocation induced by the presence of oxaliplatin.

\section{Endoplasmic reticulum (ER) stress}

Oxaliplatin $3 \mu \mathrm{M}$ was able to significantly increase the ER stress marker GRP78 (Figure 2D). Interestingly, only Mg treat-
$\mathbf{A}$

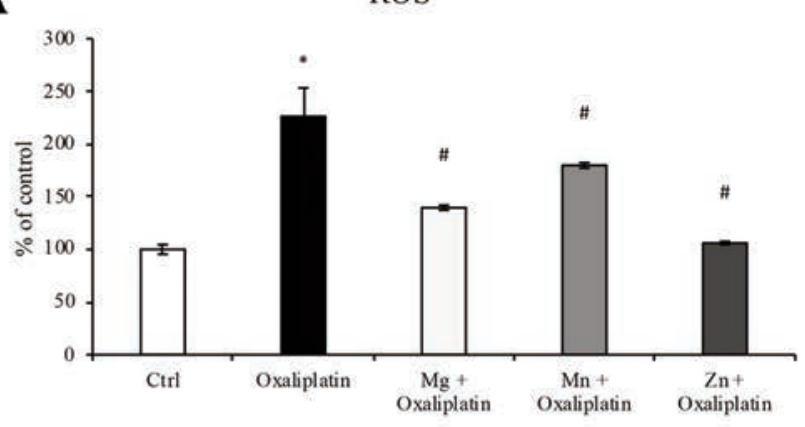

C

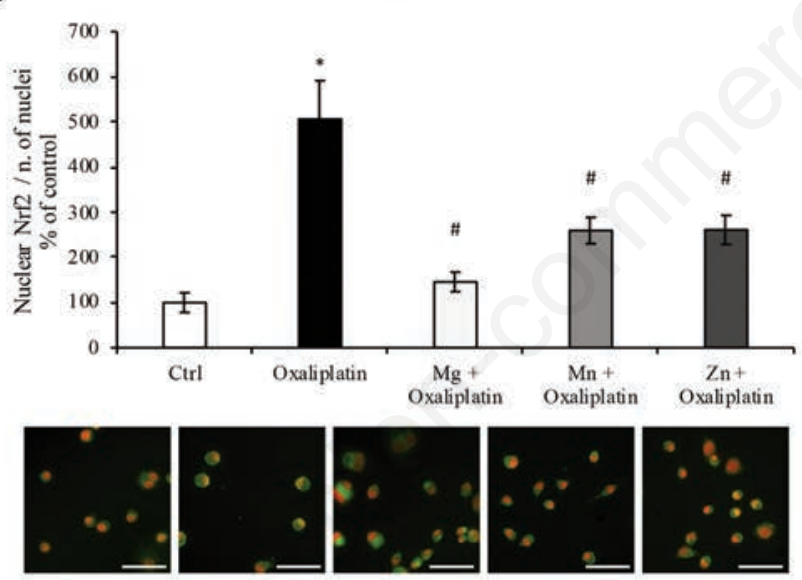

B

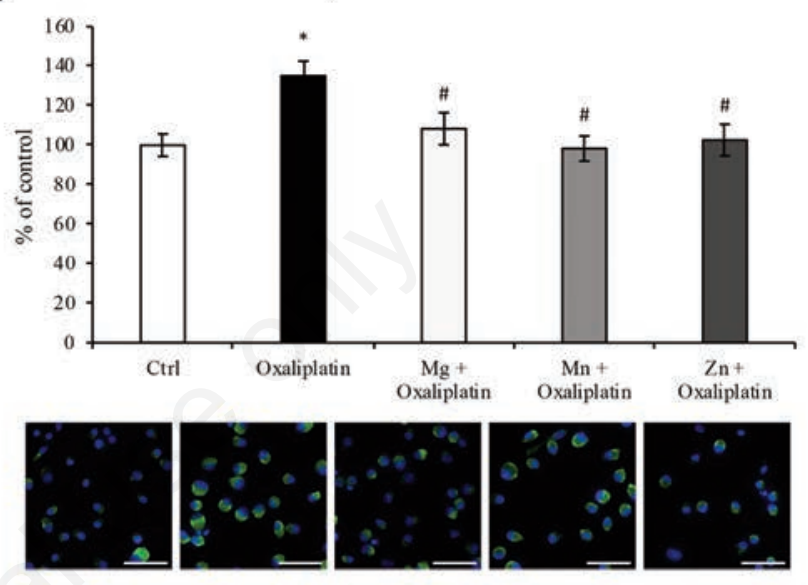

D

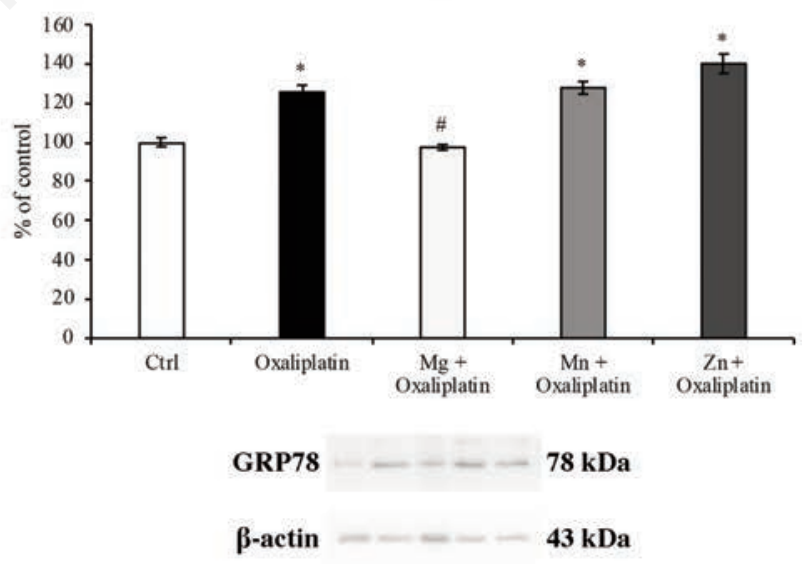

Figure 2. Oxaliplatin-induced subcellular impairment and its protective effects of micronutrients. A) The ROS were evaluated in order to highlight the role of oxaliplatin to induce oxidative stress; microglial BV-2 cells increased the ROS production during oxaliplatin treatment; such an increase in ROS overproduction was counteracted by the presence of micronutrients in the cell medium; values are expressed in percentage of control (Ctrl, untreated cells) as mean \pm SEM; “* ${ }^{*}<0.05$ vs $\mathrm{Ctrl}$; \#p $<0.05$ vs oxaliplatin. Each experiment was performed in triplicate, for three different experimental setups. B) Immunofluorescent analysis of cytochrome $\mathrm{C}$ shown a significant cytoplasmic increase after $24 \mathrm{~h}$ of $3 \mu \mathrm{M}$ oxaliplatin treatment (oxaliplatin); on the other hand, pre- and co-treatment of Mg $1 \mathrm{mM}$, Mn $50 \mathrm{nM}$ and $\mathrm{Zn} 100 \mathrm{nM} 1 \mathrm{mM} \mathrm{Mg}, 50 \mathrm{nM}$ Mn and $100 \mathrm{nM} \mathrm{Zn}$ (grey columns and last three images on the right) were able to prevent this incremen. Five microscopic fields for each experimental point were analyzed, and three different experiments were performed; blue, DAPI; green, Cytochrome C. Total magnification 200x; scale bar: $50 \mu \mathrm{m}$. Values are expressed in percentage of control (Ctrl, untreated cells) as mean \pm SEM; ${ }^{*} \mathrm{p}<0.05$ vs $\mathrm{Ctrl}$; \# $\mathbf{p}<0.05$ vs oxaliplatin. C) The Nrf2 analysis revealed a consistent nuclear translocation when BV-2 cells were treated with $3 \mu \mathrm{M}$ oxaliplatin alone for $24 \mathrm{~h}$; on the contrary, when $\mathrm{Mg}$, $\mathrm{Mn}$ and $\mathrm{Zn}$ were added in the medium, they counteract the translocation of the Nrf2 transcription factor which showed mainly a cytoplasmic localization. Five microscopic fields for each experimental point were analyzed, and three different experiments were performed; red, DAPI; green, Nrf2. Total magnification 200x; scale bar: $50 \mu \mathrm{m}$. Values are expressed in percentage of control (Ctrl, untreated cells) as mean \pm SEM; ${ }^{*} \mathrm{p}<0.05$ vs $\mathrm{Ctrl}$; $\# \mathbf{p}<0.05$ vs oxaliplatin. D) Western blotting analysis and quantification of GRP78 expression during oxaliplatin $3 \mu \mathrm{M}$ treatment at $24 \mathrm{~h}$ alone (black column) and in presence of $1 \mathrm{mM} \mathrm{Mg}$ (light grey column), $50 \mathrm{nM} \mathrm{Mn} \mathrm{(medium} \mathrm{grey} \mathrm{column)} \mathrm{or} 100 \mathrm{nM} \mathrm{Zn}$ (dark grey column), both in pre-treatment and co-treatment; values are expressed in percentage of control (Ctrl, untreated cells) as mean \pm SEM; ${ }^{*} \mathbf{p}<\mathbf{0 . 0 5}$ vs Ctrl; \# $\mathbf{p}<\mathbf{0 . 0 5}$ vs oxaliplatin. Each experiment was performed in triplicate, for three different experimental setups. 
ment was able to prevent this effect (Figure 2D, light grey column), whereas Mn and $\mathrm{Zn}$ showed no preventive effect for GRP78 increase (Figure 2D, medium and dark grey columns, respectively).

\section{Microglial markers expression}

In order to achieve if oxaliplatin was able to induce the activation of BV-2 cells, we evaluated the Iba1 and CD86 expression by immunofluorescent analysis. Oxaliplatin treatment was able to significantly increase both the Ibal and CD86 expression levels (Figure 3, black column and second image from left). This effect was counteracted by the presence of $\mathrm{Mg}$, Mn or $\mathrm{Zn}$ (grey columns and last three images on the right).
$\mathbf{A}$

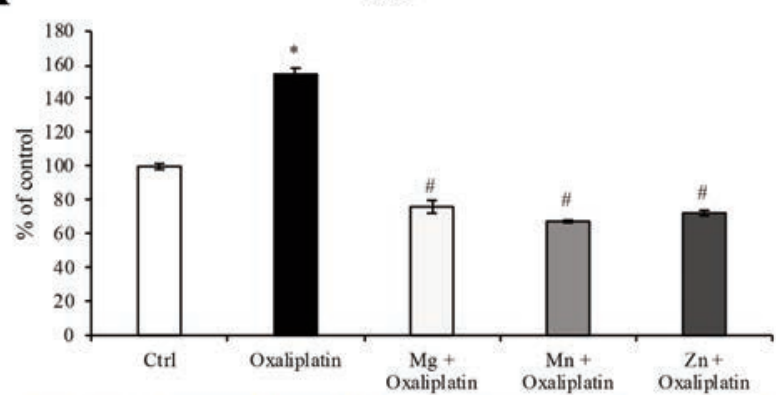

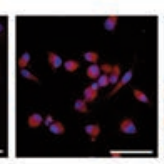

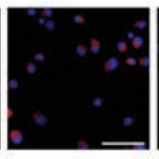

CD86

B

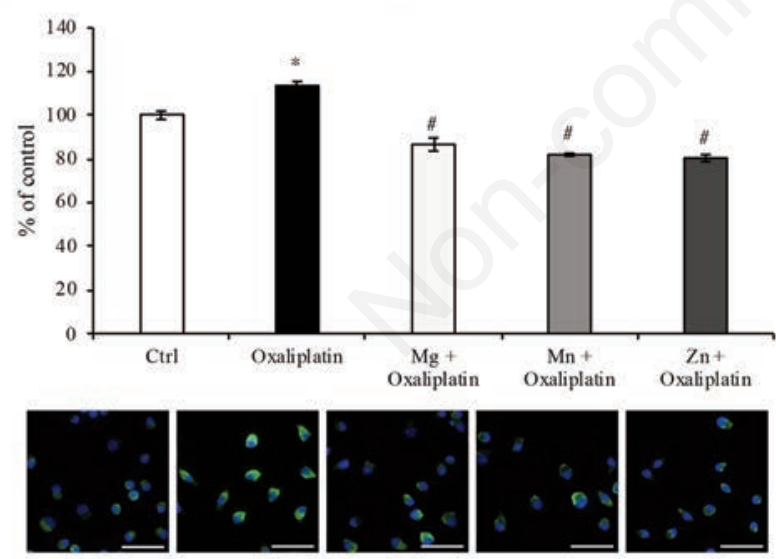

Figure 3. Microlements were able to inhibit microglial activation. Immunofluorescent analysis of Ibal (A) show a significant increase (more than $50 \%$ ) after $24 \mathrm{~h}$ of oxaliplatin $3 \mu \mathrm{M}$ treatment (black histogram and second images from the left). On the other hand, pre- and co-treatment with $1 \mathrm{mM} \mathrm{Mg}, 50 \mathrm{nM} \mathrm{Mn}$ and $100 \mathrm{nM} \mathrm{Zn}$ (grey columns and last three images on the right) were able to prevent this increase. A similar result was obtained for the analysis of CD86 (B). Five microscopic fields for each experimental point were analyzed, and three different experiments were performed; blue, DAPI; red, Iba1; green, CD86. Total magnification 200x; scale bar: $50 \mu \mathrm{m}$. Values are expressed in percentage of control (Ctrl, untreated cells) as mean \pm SEM; ${ }^{*} \mathbf{p}<0.05$ vs Ctrl; \# $\mathbf{p}<0.05$ vs oxaliplatin.

\section{Discussion and Conclusions}

Despite its efficacy in counteracting cancer development, oxaliplatin is associated to the onset of neuropathic pain as its negative interaction with glial cells has been widely demonstrated in vivo. Although the activation of microglia during oxaliplatininduced neuropathic pain is still much debated, ${ }^{15}$ many studies showed that both microglia and astrocytes are activated in the early phase of oxaliplatin treatment. .,6,16-18 $^{-1}$

Recent studies have shown that the main cause of the onset of neuropathy is an oxaliplatin-dependent production of ROS. ${ }^{19}$ Since activated microglia is a major sources of $\operatorname{ROS},{ }^{20}$ we tried to delineate, albeit partially, the signaling pathway that is triggered in microglial cells treated with oxaliplatin. The oxaliplatin concentration was chosen to be comparable to that administered to patients undergoing chemotherapy and suffering from chronic neuropathy. ${ }^{14}$ The concentration of $\mathrm{Mg}, \mathrm{Mn}$ and $\mathrm{Zn}$ were used as previously reported. ${ }^{21-23}$

Our results clearly demonstrate that oxaliplatin induced a ROS and cytoplasmic cytochrome $\mathrm{C}$ significant increase paralleled by a significant increase in GRP78 expression levels in BV-2 cell line. It has been demonstrated that the ER stress is linked to oxidative stress and inflammatory signalling pathway, ${ }^{24}$ thus leading to glial activation. ${ }^{25}$ Indeed, as shown by immunofluorescence analysis, oxaliplatin increased the number of microglia activated cells, showing an upregulation of Iba1, an activation marker, ${ }^{26}$ and CD86, a pro-inflammatory marker. ${ }^{27}$ This microglia polarization towards an inflammatory phenotype is further corroborated by the increase in the nuclear localization of $\mathrm{Nrf} 2$, a transcriptional factor that induces the transcription of many antioxidant genes, protecting cells from inflammation. ${ }^{28}$

All these side effects promoted by oxaliplatin were counteracted by the presence of $\mathrm{Mg}, \mathrm{Mn}$ or $\mathrm{Zn}$ elements, known to play a role in the cellular antioxidant defences.

Interestingly, the oxaliplatin-dependent ER stress was only prevented by $\mathrm{Mg}$ treatment. This data is in agreement with those of other authors demonstrating that a $\mathrm{Mg}$ deficiency suppress the Nrf2 nuclear translocation, thus decreasing antioxidant enzyme activity. ${ }^{29,30}$

In conclusion, our findings demonstrate in vitro microglial activation after oxaliplatin treatment and elucidate some of the molecular mechanisms involved in this signaling pathway. Even if further investigations are needed to better delineate this molecular pathway and although the efficacy of microelements in the treatment of neuropathy is still debated, the present data demonstrate that these metals are effective in preventing microglial oxidative stress that in turn induces neurotoxicity.

\section{References}

1. Kanavos P. The rising burden of cancer in the developing world. Ann Oncol 2006;17:viii15-23.

2. Bray F, Ferlay J, Soerjomataram I, Siegel RL, Torre LA, Jemal A. Global cancer statistics 2018: GLOBOCAN estimates of incidence and mortality worldwide for 36 cancers in 185 countries. CA Cancer J Clin 2018;68:394-424.

3. Alcindor T, Beauger N. Oxaliplatin: a review in the era of molecularly targeted therapy. Curr Oncol 2011;18:18-25.

4. Fischer J, Ganellin CR. Analogue-based drug discovery. Weinheim, Wiley-VCH; 2006.

5. Di Cesare Mannelli L, Pacini A, Micheli L, Tani A, Zanardelli M, Ghelardini C. Glial role in oxaliplatin-induced neuropathic pain. Exp Neurol 2014;261:22-33. 
6. Di Cesare Mannelli L, Pacini A, Bonaccini L, Zanardelli M, Mello T, Ghelardini C. Morphologic Features and glial activation in rat oxaliplatin-dependent neuropathic pain. J Pain 2013;14:1585-600.

7. Branca JJV, Maresca M, Morucci G, Becatti M, Paternostro F, Gulisano M, et al. Oxaliplatin-induced blood brain barrier loosening: a new point of view on chemotherapy-induced neurotoxicity. Oncotarget 2018;9:23426-38.

8. Stankovic JSK, Selakovic D, Mihailovic V, Rosic G. Antioxidant supplementation in the treatment of neurotoxicity induced by platinum-based chemotherapeutics - A review. Int J Mol Sci 2020;26:7753.

9. Pickering G, Morel V, Simen E, Cardot J-M, Moustafa F, Delage $\mathrm{N}$, et al. Oral magnesium treatment in patients with neuropathic pain: a randomized clinical trial. Magnes Res 2011;24:28-35.

10. Visovsky C. Calcium and magnesium for oxaliplatin-induced neurotoxicity: Issues in study design, measurement, and analysis. J Adv Pract Oncol 2015;6:272-6.

11. Bocchi L, Branca JJV, Pacini S, Cosentino A, Morucci G, Ruggiero M. Effect of ultrasounds on neurons and microglia: Cell viability and automatic analysis of cell morphology. Biomed Signal Proc 2015;22:44-53.

12. Branca JJV, Morucci G, Becatti M, Carrino D, Ghelardini C, Gulisano M, et al. Cannabidiol protects dopaminergic neuronal cells from cadmium. Int $\mathrm{J}$ Environ Res Public Health 2019;16:4420.

13. Carrino D, Branca JJV, Becatti M, Paternostro F, Morucci G, Gulisano M, et al. Alcohol-induced blood-brain barrier impairment: An in vitro study. Int J Environ Res Public Health 2021;18:2683.

14. Di Cesare Mannelli L, Zanardelli M, Failli P, Ghelardini C. Oxaliplatin-induced oxidative stress in nervous systemderived cellular models: Could it correlate with in vivo neuropathy? Free Radic Biol Med 2013;61:143-50.

15. Fumagalli G, Monza L, Cavaletti G, Rigolio R, Meregalli C. Neuroinflammatory process involved in different preclinical models of chemotherapy-induced peripheral neuropathy. Front Immunol 2021;11:626687.

16. Di Cesare Mannelli L, Pacini A, Corti F, Boccella S, Luongo L, Esposito E, et al. Antineuropathic profile of N-palmitoylethanolamine in a rat model of oxaliplatin-induced neurotoxicity. PLoS One 2015;10:e0128080.

17. Di Cesare Mannelli L, Pacini A, Matera C, Zanardelli M, Mello T, De Amici M, et al. Involvement of $\alpha 7 \mathrm{nAChR}$ subtype in rat oxaliplatin-induced neuropathy: Effects of selective acti- vation. Neuropharmacology 2014;79:37-48.

18. Micheli L, Mattoli L, Maidecchi A, Pacini A, Ghelardini C, Di Cesare Mannelli L. Effect of Vitis vinifera hydroalcoholic extract against oxaliplatin neurotoxicity: in vitro and in vivo evidence. Sci Rep 2018;8:14364.

19. Shim HS, Bae C, Wang J, Lee K-H, Hankerd KM, Kim HK, et al. Peripheral and central oxidative stress in chemotherapyinduced neuropathic pain. Mol Pain 2019;15:17448069 1984009

20. Loane DJ, Kumar A. Microglia in the TBI brain: The good, the bad, and the dysregulated. Exp Neurol 2016;275:316-27.

21. Segawa S, Tatsumi N, Ohishi A, Nishida K, Nagasawa K. Characterization of zinc uptake by mouse primary cultured astrocytes and microglia. Metallomics 2015;7:1067-77.

22. Sun Y, Sukumaran P, Singh BB. Magnesium-induced cell survival is dependent on TRPM7 expression and function. Mol Neurobiol 2020;57:528-38.

23. Rivas-García L, Quiles JL, Varela-López A, Arredondo M, Lopez P, Diéguez AR, et al. In vitro study of the protective effect of manganese against vanadium-mediated nuclear and mitochondrial DNA damage. Food Chem Toxicol 2020;135: 110900 .

24. Hotamisligil GS. Endoplasmic reticulum stress and the inflammatory basis of metabolic disease. Cell 2010;140:900-17.

25. Sen T, Saha P, Gupta R, Foley LM, Jiang T, Abakumova OS, et al. Aberrant ER stress induced neuronal-IFN $\beta$ elicits white matter injury due to microglial activation and T-cell infiltration after TBI. J Neurosci 2020;40:424-46.

26. Hovens I, Nyakas C, Schoemaker R. A novel method for evaluating microglial activation using ionized calcium-binding adaptor protein-1 staining: cell body to cell size ratio. Neuroimmunol Neuroinflam 2014;1:82.

27. Jurga AM, Paleczna M, Kuter KZ. Overview of general and discriminating markers of differential microglia phenotypes. Front Cell Neurosci 2020 6;14:198.

28. Ma Q. Role of Nrf2 in oxidative stress and toxicity. Annu Rev Pharmacol Toxicol 2013;53:401-26.

29. Wei S-P, Jiang W-D, Wu P, Liu Y, Zeng Y-Y, Jiang J, et al. Dietary magnesium deficiency impaired intestinal structural integrity in grass carp (Ctenopharyngodon idella). Sci Rep 2018;8:12705.

30. Simón J, Goikoetxea-Usandizaga N, Serrano-Maciá M, Fernández-Ramos D, Sáenz de Urturi D, Gruskos JJ, et al. Magnesium accumulation upon cyclin M4 silencing activates microsomal triglyceride transfer protein improving NASH. J Hepatol 2021;75:34-45.

Received for publication: 1 June 2021. Accepted for publication: 20 October 2021.

This work is licensed under a Creative Commons Attribution-NonCommercial 4.0 International License (CC BY-NC 4.0).

(C) Copyright: the Author(s), 2021

Licensee PAGEPress, Italy

European Journal of Histochemistry 2021; 65(s1):3285

doi:10.4081/ejh.2021.3285 